\title{
Results from the Sudbury Neutrino Observatory Phase III
}

\author{
The SNO Collaboration
}

This work was supported by the Director, Office of Science, Office of Basic Energy Sciences, of the U.S. Department of Energy under Contract No. DE-AC02-05CH11231.

\section{DISCLAIMER}

This document was prepared as an account of work sponsored by the United States Government. While this document is believed to contain correct information, neither the United States Government nor any agency thereof, nor The Regents of the University of California, nor any of their employees, makes any warranty, express or implied, or assumes any legal responsibility for the accuracy, completeness, or usefulness of any information, apparatus, product, or process disclosed, or represents that its use would not infringe privately owned rights. Reference herein to any specific commercial product, process, or service by its trade name, trademark, manufacturer, or otherwise, does not necessarily constitute or imply its endorsement, recommendation, or favoring by the United States Government or any agency thereof, or The Regents of the University of California. The views and opinions of authors expressed herein do not necessarily state or reflect those of the United States Government or any agency thereof or The Regents of the University of California. 


\title{
Results from the Sudbury Neutrino Observatory Phase III
}

\author{
G. Prior ${ }^{\mathrm{a}}$ on behalf of the SNO Collaboration

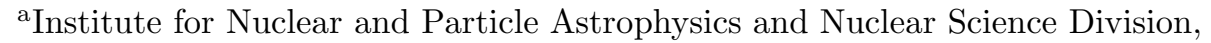 \\ Lawrence Berkeley National Laboratory, Berkeley, CA 94720, USA
}

The third and last phase of the Sudbury Neutrino Observatory (SNO) used a technique independent of previous methods, to measure the rate of neutral-current interactions in heavy water and determine precisely the total active ${ }^{8} \mathrm{~B}$ solar neutrino flux. The total flux obtained is $5.54_{-0.31}^{+0.33}$ (stat) $)_{-0.34}^{+0.36}(\mathrm{syst}) \times 10^{6} \mathrm{~cm}^{-2} \mathrm{~s}^{-1}$, in agreement with previous measurements and standard solar models. Results from a global analysis of solar and reactor neutrino give $\Delta m^{2}=7.59_{-0.21}^{+0.19} \times 10^{-5} \mathrm{eV}^{2}$ and $\theta=34.4_{-1.2}^{+1.3}$ degrees with a reduced uncertainty on the mixing angle compared to previous phases.

\section{INTRODUCTION}

\subsection{Detector}

The Sudbury Neutrino Observatory (SNO) is a 1 kton heavy water Cherenkov detector, located at a depth of $2092 \mathrm{~m}(5890 \pm 200 \mathrm{~m}$ of water equivalent) in Vale INCO's Creighton mine near Sudbury (Ontario, Canada). The detector consists of a $12 \mathrm{~m}$ diameter acrylic vessel holding the ultra-pure $\mathrm{D}_{2} \mathrm{O}$ target surrounded by 7 tons of ultra-pure $\mathrm{H}_{2} \mathrm{O}$ shielding. The Cherenkov light is recorded by 9456 inward-looking and 91 outward looking $20 \mathrm{~cm}$ diameter photo-multiplier tubes (PMTs), mounted on a stainless-steel geodesic support structure surrounding the acrylic vessel and providing an effective coverage of $59 \%$. Its primary goal is to detect solar neutrinos but it allows also the measurement of atmospheric neutrinos and muons as well as supernovae bursts search. The volume of water outside the geodesic structure provides shielding against cosmic-rays and naturally occuring radioactivity from the rock and the construction material.

\subsection{Interactions}

SNO measures the flux from ${ }^{8} \mathrm{~B}$ neutrinos in the pp-chain through three different reactions: charged-current (CC), neutral-current (NC) and elastic-scattering (ES) reactions.

In the charged-current reaction (CC):

$\nu_{e}+d \rightarrow p+p+e^{-}$ the electron energy is well correlated with the energy of the incoming electron neutrino, the unique flavor participating to this reaction.

The neutral-current reaction (NC):

$\nu_{x}+d \rightarrow p+n+\nu_{x}$

in which all the neutrino flavors participate equally, provides a measurement of the total neutrino flux above the energy threshold of $2.2 \mathrm{MeV}$. The elastic scattering reaction (ES):

$\nu_{x}+e^{-} \rightarrow \nu_{x}+e^{-}$

is sensitive to all flavors but with a reduced sensitivity to $\nu_{\mu}$ and $\nu_{\tau}$. It provides directional information on the incoming neutrino.

The CC and ES reactions are observed by detecting the Cherenkov light produced by the electron in the final state, whereas for the neutral-current reaction the neutron capture on a nucleus will be used as the signal.

\subsection{Data set}

Three different methods have been used for the neutron capture from $\mathrm{NC}$ reactions and the analyses of those data have been broken down in three phases. In the first phase, neutrons from the $\mathrm{NC}$ reaction were detected by the Cherenkov light produced by the interaction of the $6.25 \mathrm{MeV} \gamma$ ray emitted in a neutron capture on deuterium with a cross-section of $0.5 \mathrm{mb}$ and a $14 \%$ detection efficiency. The significant difference between 
the CC and NC flux obtained from this phase was the first direct evidence for neutrino flavor transformation.

In the second phase, two tons of ultra-pure salt $(\mathrm{NaCl})$ were added to the heavy water to enhance the neutron capture with a cross-section of $44 \mathrm{~b}$. The neutron capture on the ${ }^{35} \mathrm{Cl}$ results in the emission of multiple $\gamma$-rays at $8.6 \mathrm{MeV}$ threshold and with a capture efficiency of $41 \%$.

In the third and final phase of SNO, the neutron produced by the $\mathrm{NC}$ reaction have been captured by the ${ }^{3} \mathrm{He}$-gas of an array of proportional counters called Neutral Current Detectors (NCDs) with a much higher capture cross-section of $5330 \mathrm{~b}$ but a limited efficiency of $21 \%$. SNO construction started in 1997 and the data-taking ended in November 2006 with 306.4 live days of data for the $\mathrm{D}_{2} \mathrm{O}$ phase [1], 391.4 live days of data for the salt phase [2] and 385.2 live days of data for the NCD phase [3].

\section{NEUTRAL CURRENT DETECTORS}

\subsection{Motivations}

The idea of deploying a new set of detector for the SNO third phase is three fold. It offers the advantage to produce different systematics compared to previous phases. A better measurement of the CC flux can be done since the neutron from the $\mathrm{NC}$ reaction will capture predominently on the ${ }^{3} \mathrm{He}$ of the counters, as a consequence the background to the CC signal from the $6.25 \mathrm{MeV}$ $\gamma$-ray from neutron capture on deuterium will be greatly reduced. Finally by allowing the NCD array to detect most of the $\mathrm{NC}$ reaction the correlations between measurements of the $\mathrm{CC}$ and $\mathrm{NC}$ fluxes are highly reduced.

\subsection{Detector}

The Neutral Current Detectors (NCDs) consist of an ultra-low radioactivity proportional array of 36 counters filled with a mixture of ${ }^{3} \mathrm{He}$ and $\mathrm{CF}_{4}$ gas and 4 additional counters filled with a mixture of ${ }^{4} \mathrm{He}$ and $\mathrm{CF}_{4}$ gas used for background measurements. The counters were deployed vertically in the $\mathrm{D}_{2} \mathrm{O}$ on a $1 \times 1 \mathrm{~m}$ grid. The total deployed array length is $398 \mathrm{~m}$. The walls of the counters are made of $360 \mu \mathrm{m}$ thick ultra-pure chemically vapor deposited nickel. Measurements of the content of uranium and thorium in the $\mathrm{Ni}$ walls gives $3.43_{-2.11}^{+1.49} \times 10^{-12} \mathrm{gTh} / \mathrm{gNi}$ and $1.81_{-1.12}^{+0.80} \times 10^{-12}$ $\mathrm{gU} / \mathrm{gNi}$, making those counters 100 times more pure than any previously existing counter of this type.

The NCD electronics have two independently triggered readout systems: a multiplexed digitizing path to allow pulse-shape discrimination to distinguish neutron-capture signal from alpha background and a fast data path using shapersADCs for collection of information at $\mathrm{kHz}$ event rate such as a supernova.

\subsection{Neutron capture efficiency}

Two different methods have been used to measure the NC neutron capture efficiency. In a first method, ${ }^{24} \mathrm{Na}\left(\mathrm{t}_{1 / 2}=14.959\right.$ hours $)$ in form of active $\mathrm{NaCl}$ has been mixed in the heavy water. The deuterium photodisintegration induced by the $2.754-\mathrm{MeV} \gamma$ from ${ }^{24} \mathrm{Na}$, produced neutrons that were captured by the NCD array. The neutron capture efficiency for this array using this method was $0.211 \pm 0.007$.

Another method used $\mathrm{AmBe}$ and ${ }^{252} \mathrm{Cf}$ neutrons point sources data to calibrate the Monte-Carlo simulation. The $\gamma \mathrm{s}$ produced by ${ }^{241} \mathrm{Am}$ hit the ${ }^{9} \mathrm{Be}$ target and produce neutrons in one case. In the other case, the fission of ${ }^{252} \mathrm{Cf}$ produces neutrons. The Monte-Carlo gave an efficiency of $0.210 \pm 0.003$ in good agreement with the value obtained from the ${ }^{24} \mathrm{Na}$ source.

\subsection{Neutron background}

Several sources of neutron background have been identified and the number of events reconstructed in the photo-multipliers (PMTs) and in the NCDs has been determined. Low levels of ${ }^{214} \mathrm{Bi}$ and ${ }^{208} \mathrm{Tl}$ are present in the heavy water, light water, NCD counters and cables. Deuteron photodisintegration and low-energy Cherenkov events from $\beta-\gamma$ decays can create free neutrons that will constitute a background to the signal. In addition alphas from Ra progeny on the NCD tube surfaces can induce ${ }^{17} \mathrm{O}(\alpha, \mathrm{n})$ and ${ }^{18} \mathrm{O}(\alpha, \mathrm{n})$ reactions. An in situ analysis of the Cherenkow light found three "hotspots" of radioactivity on two strings and the number of background neu- 
Table 1

Background events for the PMT and NCD arrays

\begin{tabular}{lll}
\hline Source & PMT events & NCD events \\
\hline $\mathrm{D}_{2} \mathrm{O}$ radioactivity & $7.6 \pm 1.2$ & $28.7 \pm 4.7$ \\
$\mathrm{NCD}$ bulk $/{ }^{17} \mathrm{O},{ }^{18} \mathrm{O}$ & $4.6_{-1.6}^{+2.1}$ & $27.6_{-10.3}^{+12.9}$ \\
Atmospheric $\nu /{ }^{16} \mathrm{~N}$ & $24.7 \pm 4.6$ & $13.6 \pm 2.7$ \\
"Other backgrounds" & $0.7 \pm 0.1$ & $2.3 \pm 0.3$ \\
$\mathrm{NCD}$ "hotspots" & $17.7 \pm 1.8$ & $64.4 \pm 6.4$ \\
$\mathrm{NCD}$ cables & $1.1 \pm 1.0$ & $8.0 \pm 5.2$ \\
\hline Total internal neutron background & $56.4_{-5.4}^{+5.6}$ & $144.6_{-14.8}^{+13.8}$ \\
\hline External-source neutrons & $20.6 \pm 10.4$ & $40.9 \pm 20.6$ \\
\hline Cherenkov events from $\beta-\gamma$ decays & $5.8_{-2.9}^{+9.7}$ & - \\
\hline IAVB & $<0.3(68 \%$ C.L. $)$ & - \\
\hline
\end{tabular}

trons produced from deuterium photodisintegration due to this elevated radioactivity has been estimated. The neutron background from atmospheric neutrino interactions was also evaluated. Finally from previous phases isotropic acrylic vessel background (IAVB) events were identified and their number after data reduction for this phase has been estimated. The numbers of background events from the different sources are summarized in Table 1. Geo-neutrinos, anti-neutrinos from reactors, spontaneous fission, cosmogenics, $\mathrm{CNO}$ neutrinos and $(\alpha, \mathrm{n})$ reactions are included in "Other backgrounds".

\subsection{Instrumental backgrounds}

In addition to the neutron background from physics events, one string had a gain instability, two strings had events from electrical microdischarge, two strings had a problem of electrical connection and one of them had a gas leak into counter inter-space. Since some strings had more than one problem, it is only a total of 6 strings that have been removed from the analysis including the two strings with "hotspots" neutrons.

\subsection{Alphas background}

A detailed Monte Carlo simulation has been performed in order to evaluate the fraction of alpha events falling in the neutron energy range analysis window of $0.4-1.4 \mathrm{MeV}$. The energy deposition, electron drift, charge multiplication, ion drift, pulse propagation, modelisation of the electronics and noise have been simulated. Alphas events from polonium on the NCD wall, NCD anode wire, NCD end-cap as well as from uranium and thorium on the NCD walls and NCD anode wire have been simulated and compared well with data in the energy region from $1 \mathrm{MeV}$ to $8 \mathrm{MeV}$. Their distribution as a function of the energy served in the fit to the NCD signal.

\subsection{Challenges}

Beside the constraints on the material purity required by the introduction of a new detector in the $\mathrm{D}_{2} \mathrm{O}$, the shadowing of the NCD array onto the PMTs had to be described in a detailed way and about $10 \%$ light was lost in total.

The reduced efficiency from the neutron capture onto the ${ }^{3} \mathrm{He}$ gas provides an average detection of about 1000 neutrons per year.

\section{RESULTS}

The data presented here were recorded between November 27, 2004 and November 28, 2006. A blind analysis procedure was used to minimize the possibility to introduce biases. The first month of neutrino data remained open. The blindness scheme transformed the data set thereafter: a fraction of the final data set was hidden and a fraction of neutron events from muons interactions was added. The fraction numbers remained 
Table 2

Number of PMTs and NCDs events

\begin{tabular}{ll}
\hline PMT CC events & $1867_{-101}^{+91}$ \\
PMT ES events & $171_{-22}^{+24}$ \\
PMT NC events & $267_{-22}^{+24}$ \\
PMT background events & $77_{-10}^{+12}$ \\
\hline NCD NC events & $983_{-76}^{+77}$ \\
NCD background events & $185_{-22}^{+25}$ \\
\hline
\end{tabular}

unknown until the removal of the blindness constraint. The blindess was lifted only after analysis procedure, parameters and background were finalized.

A simultaneous fit to the number of $\mathrm{NC}$ events in the NCDs and the number of $\mathrm{NC}, \mathrm{CC}$ and ES events in the PMTs as well as the number of various background type events was made. A Markov-chain Monte Carlo (MCMC) method with the Metropolis-Hasting algorithm was employed to make the fit. In this log-likelihood fit method, the physics parameters, such as the fluxes, are fit allowing the nuisance parameters, such as the systematics weighted by external constraints, to vary.

For the NCDs, the event energy distribution was fit with an alpha background distribution, a neutron calibration spectrum, the expected neutron background and two instrumental background event distributions that had been observed in larger numbers in two of the rejected NCDs. The event energy distribution and the fit results are shown in Figure 1.

The PMTs events were fit in reconstructed kinetic energy, the cosine of the event direction with respect to the sun $\left(\cos \Theta_{\text {sun }}\right)$ and the normalized radial position $\rho^{3}=(R / 600 \mathrm{~cm})^{3}$ where $\mathrm{R}$ is the radius of the event vertex.

Two independent analysis methods were used as checks of the MCMC method. They both used maximum liklihood fits but handled the systematics in a different way. A comparison of the results from the three analysis methods after box opening on May 2nd 2008, revealed two issues. A $10 \%$ difference in the NC flux uncertainties was found and traced to an error in the input parameters in two methods. After correction of the in-

Figure 1. NCD energy spectrum fit. Data are shown after data reduction up to $1.4 \mathrm{MeV}$ and the fit is above $0.4 \mathrm{MeV}$.

puts the errors agreed and the fitted central values were unchanged. A low ES flux $(0.5 \sigma$ lower than the two other analyses) from the MCMC fit was due to a parametrization failure of the algorithm used to fit the peak of the ES posterior distribution. A more robust algorithm was implemented and the ES flux agreed well with results from the other two analyses.

The spectral distribution of the ES and CC events are not constrained to the ${ }^{8} \mathrm{~B}$ shape, they have been extracted from the data. The number of events from the fit to the data is summarized in Table 2. The equivalent neutrino flux derived from the fitted CC, ES and NC events assuming the ${ }^{8} \mathrm{~B}$ neutrino spectrum from [4] are (in units 
of $\left.10^{6} \mathrm{~cm}^{-2} \mathrm{~s}^{-1}\right)$ :

$\Phi_{C C}^{S N O}=1.67_{-0.04}^{+0.05}(\text { stat })_{-0.08}^{+0.07}($ syst $)$

$\Phi_{E S}^{S N O}=1.77_{-0.21}^{+0.24}(\text { stat })_{-0.10}^{+0.09}($ syst $)$

$\Phi_{N C}^{S N O}=5.54_{-0.31}^{+0.33}(\text { stat })_{-0.34}^{+0.36}($ syst $)$

The lower ES flux obtained here compared to the one found by Super-Kamiokande [5] is consistent with a downward statistical fluctuation in the ES signal due to a shortfall of signal in two energy bins near $\cos \left(\Theta_{\text {sun }}\right)=1$. The CC, ES and NC flux results reported here are in agreement with the $\mathrm{NC}$ flux result from the $\mathrm{D}_{2} \mathrm{O}$ [1] and fluxes from the salt phase [2] with a p-value of 0.328 .

A combined $\chi^{2}$ fit to SNO $\mathrm{D}_{2} \mathrm{O}$ and salt phases day and night energy spectra and the NCDphase data using two-flavor active neutrino models to predict the CC, $\mathrm{NC}$ and $\mathrm{ES}$ rates in SNO, yields the allowed $\Delta m^{2}$ and $\tan ^{2} \theta$ regions shown in Figure 2. In a global analysis of all solar neutrino data (including Borexino and SuperKamiokande-I), the allowed region is shown in Figure 3(b). The allowed region for the global solar analysis combined with the 2881 ton-year KamLAND reactor antineutrino results [6], is shown in Figure 3(c). The best-fit point to the

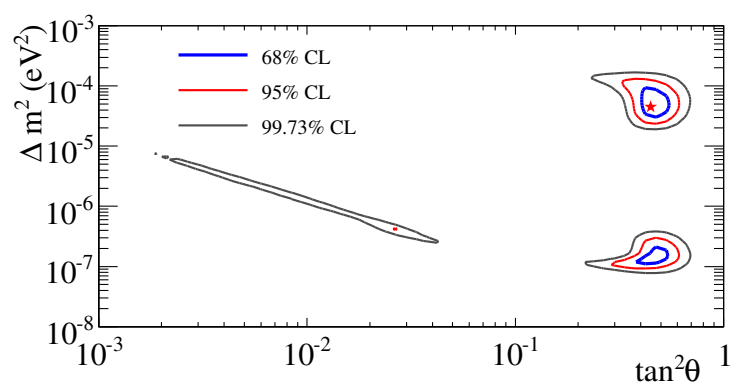

Figure 2. SNO only: $\mathrm{D}_{2} \mathrm{O} \&$ salt day and night spectra, NCD phase fluxes.

solar global plus KamLAND data yields $\Delta m^{2}=$ $7.59_{-0.21}^{+0.19} \times 10^{-5} \mathrm{eV}^{2}$ and $\theta=34.4_{-1.2}^{+1.3}$ degrees.
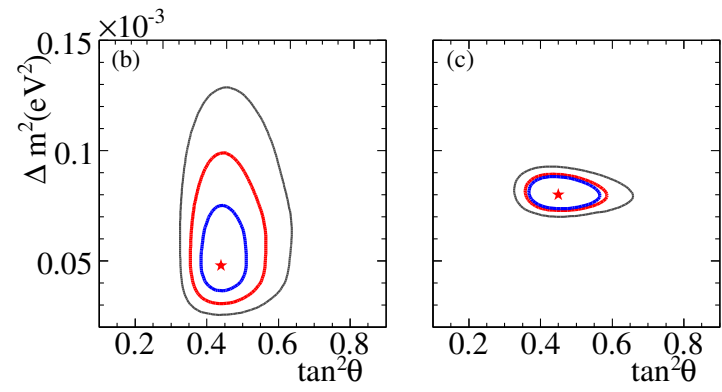

Figure 3. (b) Solar Global: SNO, SK, Cl , Ga, Borexino. (c) Solar Global + KamLAND

\section{CONCLUSION}

The Sudbury Neutrino Observatory (SNO) experiment precisely measured the total flux of active ${ }^{8} \mathrm{~B}$ neutrinos from the sun idenpendently from the previous methods used in phases I and II. The results from the NCD phase agree well with previous measurements from SNO and the correlations between fluxes from $\mathrm{CC}$ and $\mathrm{NC}$ have been reduced. The uncertainty on the mixing angle has been reduced by about $40 \%$.

\section{REFERENCES}

1. Q.R. Ahmad et al. (SNO Collaboration), Phys. Rev. Lett. 89, 011301 (2002)

2. B. Aharmim et al. (SNO Collaboration), Phys. Rev. C 72, 055502 (2005)

3. B. Aharmim et al. (SNO Collaboration), Phys. Rev. Lett. 111301 (2008)

4. W.T. Winter et al. , Phys. Rec. C 73, 025503 (2006)

5. J. Hosaka et al. , Phys. Rev. D 73, 112001 (2006)

6. S. Abe et al., Phys. Rev. Lett. 100, 221803 (2008) 